\title{
Pediatric Robotic Transplant Ureteral Reimplantation for Vesicoureteral Reflux
}

\author{
Jonathan Corbett, MD, ${ }^{1}$ and Paul H. Noh, MD²
}

\begin{abstract}
Background: The first published report of a pediatric robotic extravesical transplant ureteral reimplantation for vesicoureteral reflux (VUR) in a renal allograft is described.

Case Presentation: The patient is an 11-year-old Caucasian girl who had acute allograft pyelonephritis and was subsequently found to have dilating VUR.

Conclusion: Robotic surgery facilitated an effective nondismembered extravesical reimplant with minimal morbidity.
\end{abstract}

Keywords: kidney transplant, vesicoureteral reflux, robotic reimplant, pyelonephritis

\section{Introduction}

U urgical correction of transplant allograft vesicourOeteral reflux (VUR) has been reported, with $3.58 \%$ of patients requiring correction in one series. ${ }^{1}$ Several different surgical techniques have been reported in the correction of transplant VUR. Pediatric robotic extravesical ureteral reimplantation has been shown to be a safe and effective method of VUR correction in native kidneys, ${ }^{2,3}$ but to the best of our knowledge, there have been no published reports of this approach to correct VUR in a pediatric transplant allograft. Effective treatment utilizing robotic surgery for pediatric transplant VUR is described.

\section{Case}

An 11-year-old girl, with a history of deceased donor kidney transplantation at 6 years of age for focal segmental glomerulosclerosis, was seen after diagnosis of VUR into her transplant kidney. A nonrefluxing reimplantation of the ureter was described in the transplantation operative report. She developed acute pyelonephritis after being off of urinary tract antibiotic prophylaxis, because of a history of recurrent urinary tract infections, with allograft pain and fevers. An ultrasonography of her transplant was obtained, which showed mild urothelial thickening in the renal pelvis. Urine culture grew $>100,000 \mathrm{cfu} / \mathrm{mL}$ of Escherichia coli. The patient was initially treated with intravenous ciprofloxacin and was transitioned to cefdinir to complete a 14-day course of antibiotics.

She improved rapidly with antibiotics and was discharged home on hospital day 2. After return to her usual state of health, a voiding cystourethrogram (VCUG) was obtained, which revealed grade 3 VUR into the transplant kidney. There was no reflux into the native ureters. The native left kidney had been removed before kidney transplantation. The findings, detrimental effects of pyelonephritis, and risks and benefits of the treatment options were discussed with the family, and operative intervention for the transplant VUR was advised. A robotic extravesical ureteral reimplantation was recommended instead of gold standard open surgery because of the patient's older age, consistent with the surgeon's practice for unilateral VUR in older children who undergo reimplantation. Earlier reports on the robotic extravesical technique showed a success rate of $72 \%,{ }^{2}$ but a contemporary report with more patients had a success rate $>90 \%,{ }^{3}$ indicating there was a learning curve associated with the procedure and that it is a safe and effective option.

Cystoscopy was performed to assess the bladder and transplant ureteral orifice. The transplant ureteral orifice was identified on the right lateral dome and appeared grossly incompetent. The patient was then placed in supine position on the operating room table. A Foley catheter was placed. A daVinci Xi robotic surgical system (Intuitive Surgical, Sunnyvale, CA) was utilized for the procedure. An 8-mm robotic trocar was placed in the umbilicus using an open technique. Two additional 8-mm ports were placed, under direct vision, at the level of the umbilicus in each mid-clavicular line. The robot was docked, and the transplant kidney was readily visible in the right retroperitoneum. Small bowel adhesions over the transplant kidney were dissected off the posterior peritoneum. The transplant ureter could be seen coursing from the transplant kidney, lateral to the gonadal vessels and lateral to the right medial umbilical ligament, before entering the bladder. The peritoneum was incised

${ }^{1}$ Division of Urology, Department of Surgery, University of Cincinnati, Cincinnati, Ohio, USA.

${ }^{2}$ Division of Pediatric Urology, Cincinnati Children's Hospital Medical Center, Cincinnati, Ohio, USA. 


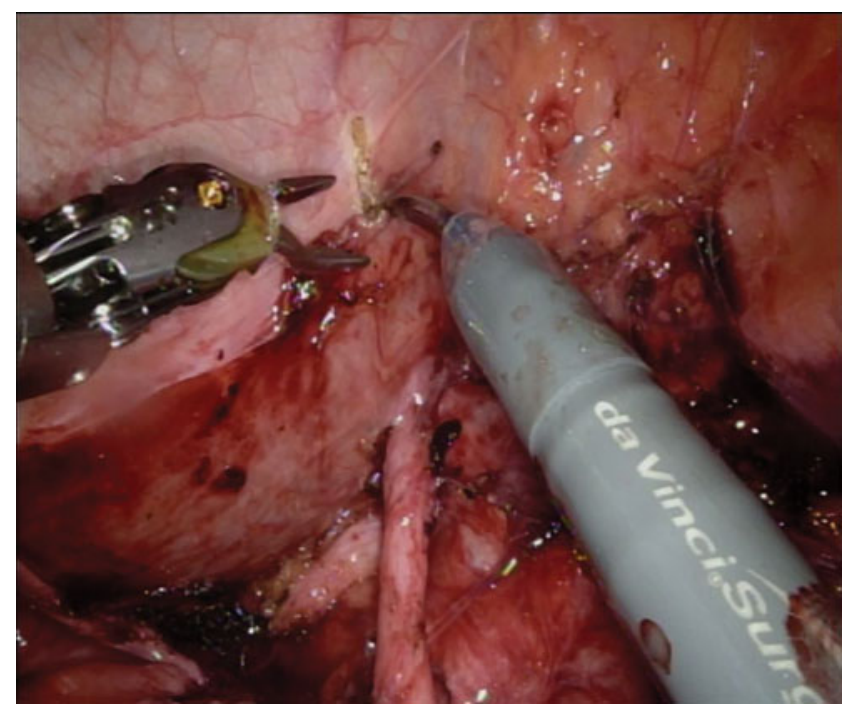

FIG. 1. Detrusor tunnel being marked.

to expose the extraperitoneal space. The right round ligament was divided to provide access to the transplant ureter. The transplant ureter was mobilized to expose the ureterovesical junction. A detrusorotomy was marked with the bladder distended to ensure a straight course for the ureter (Fig. 1). A percutaneously placed polypropylene suture was used to provide left lateral traction on the bladder from the apex of the detrusorotomy. The detrusorotomy was developed to create a trough for the transplant ureter. An inverted V-shaped dissection was performed at the ureterovesical junction, leaving the distal attachments intact. The detrusorrhaphy was performed with interrupted 3-0-polyglactin sutures, starting at the ureterovesical junction, proceeding proximally (Fig. 2). A $4 \mathrm{~cm}$ tunnel was created. The bladder was mobilized from the anterior abdominal wall and pubic bones, to minimize concern for obstruction because of a kink of the ureter at the top of the new tunnel (Fig. 3). The Foley catheter was removed before leaving the operating room. No drains were utilized. The ureter

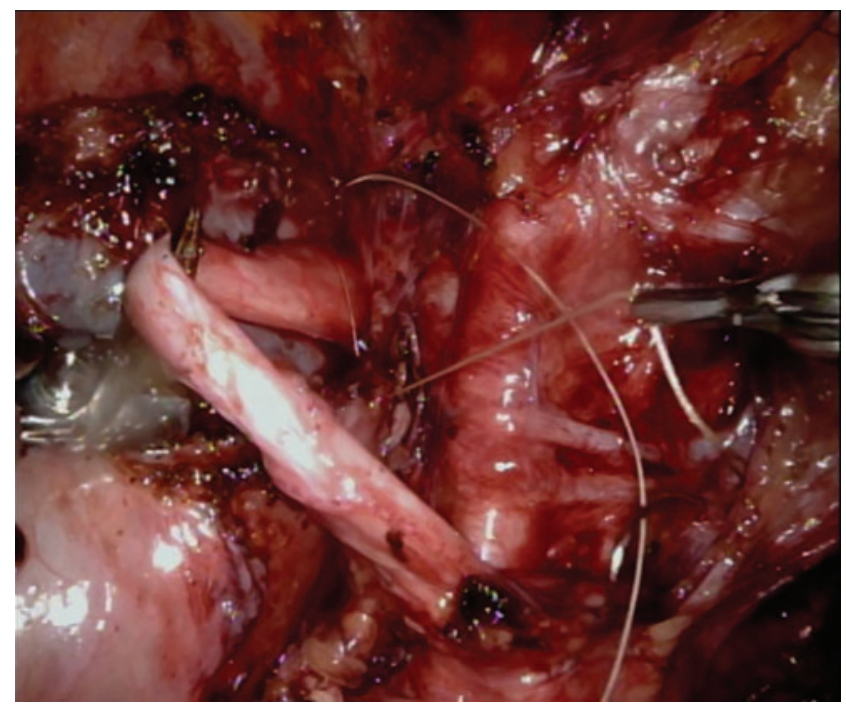

FIG. 2. Apical suture of tunnel being placed.

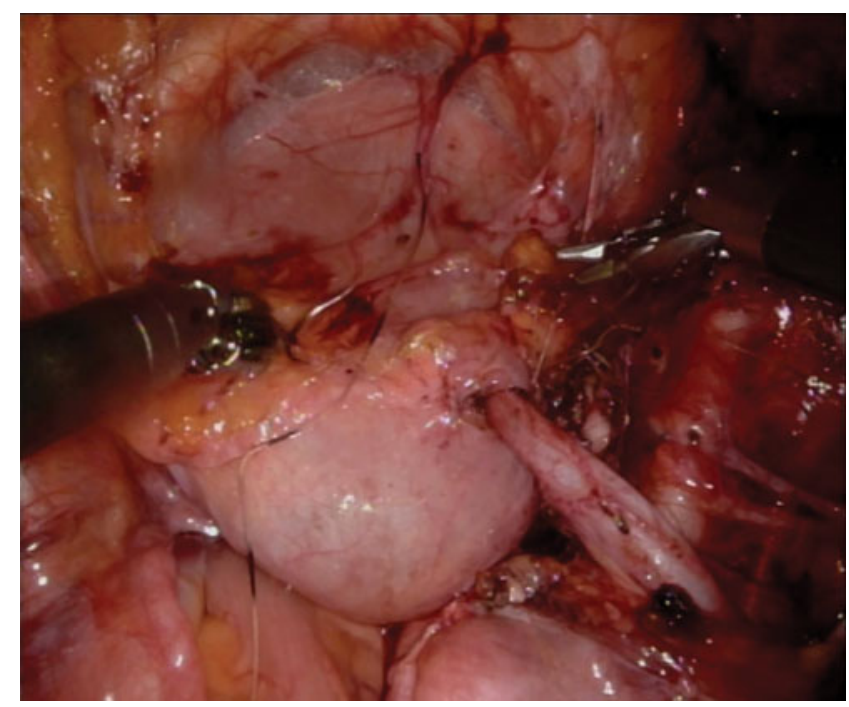

FIG. 3. Detrusor tunnel complete with bladder dropped from abdominal wall to prevent tension.

was not stented. There was no blood loss. Robotic console time was 136 minutes. No bedside assistant trocars were utilized. The patient was observed overnight and had an uneventful postoperative course with good urine output and stable renal function. No postoperative narcotic analgesia was required. The patient was discharged home on postoperative day 1 . Her outpatient recovery was uneventful. A postoperative VCUG 8 weeks after surgery showed resolution of VUR. Renal function has remained stable, and a 6-month ultrasonography showed no hydronephrosis. She has had no culture-documented urinary tract infections since the surgery.

\section{Discussion}

The prevalence of febrile urinary tract infections in children after kidney transplant ranges from $15 \%$ to $33 \% .^{4}$ VUR is a well-established risk factor for pyelonephritis. Some controversy exists over the preferred method of transplant ureteral reimplantation. A refluxing ureteral reimplantation can leave patients at risk of urinary tract infections and renal allograft damage, but offers an open channel for low-pressure drainage. A nonrefluxing ureteral reimplantation protects the transplant from VUR and risk of pyelonephritis, but leaves patients at risk for obstruction and developing high-pressure drainage.

The need for surgical correction of VUR after renal transplant appears to be low. Dinckan et al. reported on their series of 1673 transplant patients, with a $3.58 \%$ rate of surgical correction of VUR (grade 3 or higher) for recurrent febrile urinary tract infections. These patients all underwent stented open surgical correction, including an anastomosis of the transplant ureter to the native ureter in half of the patients and a reoperative dismembered ureteral reimplantation, predominantly extravesical, in half of the patients. ${ }^{1}$

Open surgical correction represents a significant morbidity for patients who have already undergone a kidney transplant. Minimally invasive approaches have the potential to reduce this morbidity. Robot-assisted laparoscopic techniques have been described to correct transplant VUR. Benamran and colleagues described their experience with robotic ureteroureterostomy for the treatment of both transplant ureteral stricture disease and 
VUR. Four of the 10 patients required conversion to open procedures because of fibrosis, but the patients who underwent robotic procedures had shorter hospital stays, less use of pain medications, and returned to a regular diet more quickly than a 7-patient cohort comparison group who underwent a planned open approach. The authors concluded that a robotic approach to post-transplant ureteral reconstruction was feasible, safe, and efficient, and offers the classical advantages of minimally invasive surgery. ${ }^{5}$

To the best of our knowledge, we report the first case of a robotic extravesical transplant ureteral reimplantation for VUR in a pediatric patient. Our patient represents another example demonstrating the benefits of robotic surgery to minimize the morbidity of surgery even in a high-risk population such as pediatric renal transplant recipients.

\section{Conclusions}

Pediatric robotic extravesical ureteral reimplantation of a transplant ureter is feasible, and can be safe and effective with minimal morbidity even in the setting of a high-risk patient.

\section{Disclosure Statement}

No competing financial interests exist.

\section{Funding Information}

No funding was received for this article.

\section{References}

1. Dinckan A, Aliosmanoglu I, Kocak H, et al. Surgical correction of vesico-ureteric reflux for recurrent febrile urinary tract infections after kidney transplantation. BJU Int 2013; E366-E371.

2. Grimsby GM, Dwyer ME, Jacobs MA, et al. Multiinstitutional review of outcomes of robotic-assisted laparo- scopic extravesical ureteral reimplantation. J Urol 2015; 193(5 Suppl):1791-1795.

3. Boysen WR, Akhavan A, Ko J, et al. Prospective multicenter study on robot-assisted laparoscopic extravesical ureteral reimplantation (RALUR-EV): Outcomes and complications. J Pediatr Urol 2018;262.e1-e262.e6.

4. John U, Kemper MJ. Urinary tract infections in children after renal transplantation. Pediatr Nephrol 2009;24:11291136.

5. Benamran DA, Klein J, Hadaya K, et al. Post-kidney transplant robot-assisted laparoscopic ureteral (donor-receiver) anastomosis for kidney graft reflux or stricture disease. Urology 2017;108:96-101.

Address correspondence to: Jonathan Corbett, $M D$ Division of Urology Department of Surgery University of Cincinnati 231 Albert Sabin Way, ML 0589 Cincinnati, $\mathrm{OH} 45267$

USA

E-mail: corbetja@ucmail.uc.edu

Cite this article as: Corbett J, Noh PH (2020) Pediatric robotic transplant ureteral reimplantation for vesicoureteral reflux, Journal of Endourology Case Reports 6:2, 96-98, DOI: 10.1089/cren.2019.0057. 